\title{
Retroauricular Hairline Approach for Excision of Second Branchial Cleft Cysts
}

\author{
${ }^{1}$ Brad LeBert, ${ }^{1}$ Sean R Weiss, ${ }^{2}$ Jonas T Johnson, ${ }^{1}$ Rohan R Walvekar \\ ${ }^{1}$ Department of Otolaryngology and Head and Neck Surgery, LSU Health Sciences Center, New Orleans, USA \\ ${ }^{2}$ Department of Otolaryngology and Head and Neck Surgery, University of Pittsburgh School of Medicine, Pittsburgh, USA
}

Correspondence: Rohan R Walvekar, Assistant Professor, Department of Otolaryngology and Head and Neck Surgery, LSU Health Sciences Center, 533 Bolivar Street, Suite 566, New Orleans 70112, LA, USA, Phone: (504) 568 4785, Fax: (504) 5684460 e-mail: rwalve@Isuhsc.edu

\begin{abstract}
Objective: To report our preliminary experience with a retroauricular hairline incision (RAHI) for excision of second branchial cleft cysts (SBCC) and to present a relevant literature review.

Study design: Retrospective case series.

Methods: A retrospective chart review was conducted of two consecutive patients diagnosed with SBCC who underwent surgical removal via a RAHI. Relevant demographic, clinicopathological and radiological data were recorded. A web-based search was conducted to identify relevant scientific literature on "retroauricular hairline incision/approach" in order to present a systematic review of current literature.

Results: In both cases, the SBCC $(6.0$ and $3.8 \mathrm{~cm})$ could be safely excised without major complications. One patient developed a temporary hypoesthesia of earlobe. None of the patients had a postoperative infection, hematoma, spillage of cyst fluid or necrosis of the skin flap. All patients were satisfied with the cosmetic outcome of the procedure $(100 \%)$. The incisions healed well without any evidence of hair loss or keloid formation.

Conclusion: In conclusion, the RAHI offers an excellent surgical outcome and superior cosmetic result with no proven increased risks to the patients who require surgical excision of a benign SBCC

Keywords: Retroauricular, Upper neck mass, Branchial cleft cyst, Lateral cervical cyst, Postauricular.
\end{abstract}

\section{INTRODUCTION}

In recent years, there has been increasing attention to cosmetic results associated with making cervical incisions for benign neck lesions. ${ }^{1-4}$ Minimally invasive techniques using either endoscopic guidance or distant access incisions have been proposed for treating benign cervical lesions. ${ }^{1-4}$ The goal of these surgical innovations is often improved cosmesis, visualization, and magnification with the endoscopic guidance. Branchial cleft anomalies are benign congenital lesions that result from incomplete obliteration of branchial clefts or pouches and represent nearly 30\% of congenital neck masses. Second branchial cleft cysts (SBCC) are the most common of these anomalies and often present anterior to the upper-third of the sternocleidomastoid muscle. ${ }^{5,6}$ SBCC are typically painless, well-circumscribed, cystic masses that frequently manifest during or shortly after an upper respiratory infection. The primary treatment for SBCC is surgical excision which has traditionally been through a cervical incision placed in a favorable horizontal skin crease overlying the lesion. Removal of upper neck masses by way of an incision placed in the postauricular sulcus and hairline also known as the retroauricular hairline incision (RAHI) has been shown to result in better cosmetic outcome and patient satisfaction compared with conventional cervical incisions. ${ }^{1}$ In our review of current English literature, we came across three articles describing the results of the retroauricular auricular approach for excision of upper neck masses and SBCC. ${ }^{1,3,7}$ Our literature search revealed that the feasibility and utility of this approach has not been discussed in western literature. We report two cases of SBCC treated surgically by way of a postauricular hairline incision approach and also discuss potential pitfalls associated with management of lateral cervical cysts.

\section{METHODS}

A retrospective chart review was conducted of two consecutive patients diagnosed with SBCC who underwent surgical removal via RAHI. The study was conducted at the Department of Otolaryngology and Head and Neck Surgery, Louisiana State University Health Sciences Center, New Orleans, LA. Relevant demographic, clinicopathological and radiological data was recorded. A web-based search was conducted to identify relevant scientific literature on retroauricular hairline incision/approach in order to present a systematic review of current literature. Keywords entered included, "retroauricular hairline approach, retroauricular 
Table 1: Retroauricular hairline approach: Surgical steps

- General anesthesia; position-supine with neck extended with a shoulder roll and head rotated to the opposite side.

- An incision is made beginning at the inferior limit of the postauricular sulcus which is then curved into the hairline first posterior and then caudally (Fig. 1)

- A postauricular flap is then elevated like in a "face-lift procedure" identifying and preserving the greater auricular nerve and the external jugular vein.

- A subplatysmal plane of dissection can be obtained via a triangle at the upper end of the neck defined by the anterior border of the sternocleidomastoid muscle, poster ramus of the mandible, and upper fibers of insertion of the platysmal muscle to the lower border of the mandible. Care must be taken to avoid injury to the marginal mandibular branch of the facial nerve by careful dissection and use of bipolar coagulation.

- The great auricular nerve can be mobilized by sharp dissection and retract as needed.

- The anterior border of sternocleidomastoid muscle is then identified and delineated as inferiorly as possible and then retracted in a posteroinferior direction. The skin flap is elevated by surgical retraction. At this point the mass can be visualized and the surgical exposure is complete (Fig. 2). If necessary, surgical exposure and maneuverability can be improved by decompressing the mass by aspirating its contents. This needle puncture should be made in a nondependent position and can be sealed with a stitch or a hemostat.

- The mass is dissected with sharp and blunt finger dissection from its attachments. Structures that need to be identified and preserved include the spinal accessory nerve, carotid sheath containing the internal jugular vein and carotid artery, posterior belly of digastrics, and hypoglossal nerve. The internal jugular vein is often compressed by the mass and is usually found posterior to it.

- Once free of its attachment, finger dissection helps the mass to be delivered into the surgical wound. A complete removal of the SBCC is accomplished by releasing its attachment to the floor of the neck and internal jugular vein.

- A surgical drain is then placed within the cavity created by the cyst removal and is fashioned to exit the skin via a small separate incision within the hairline posterior to the retroauricular incision (Fig. 3).

- The skin flap is repositioned and sutured in layers.

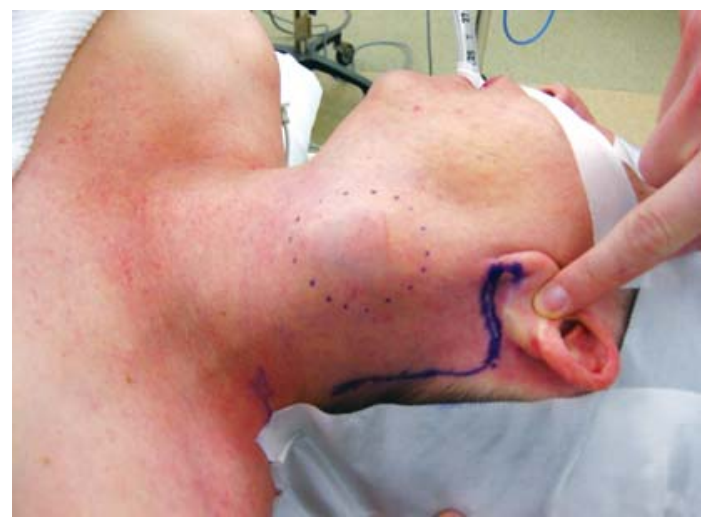

Fig. 1: Retroauricular hairline incision

hairline incision, RAHI, surgical approaches to the upper neck, branchial cleft cyst, and lateral cervical cysts”. In both cases, the neck mass was surgically removed by a RAHI

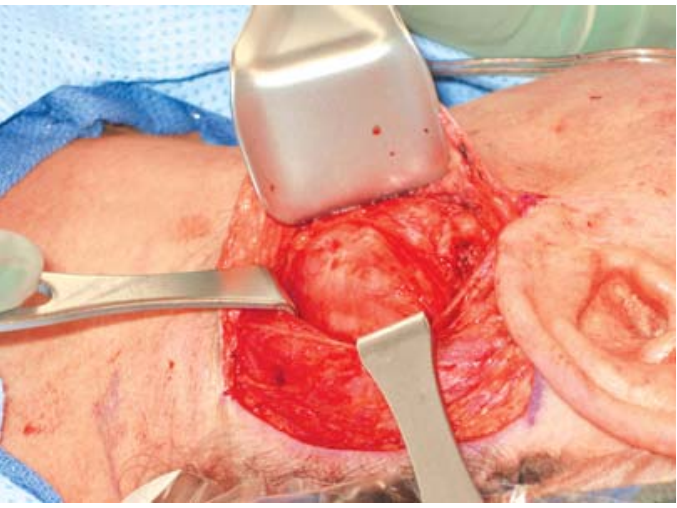

Fig. 2: Surgical exposure via a retroauricular approach to the upper neck

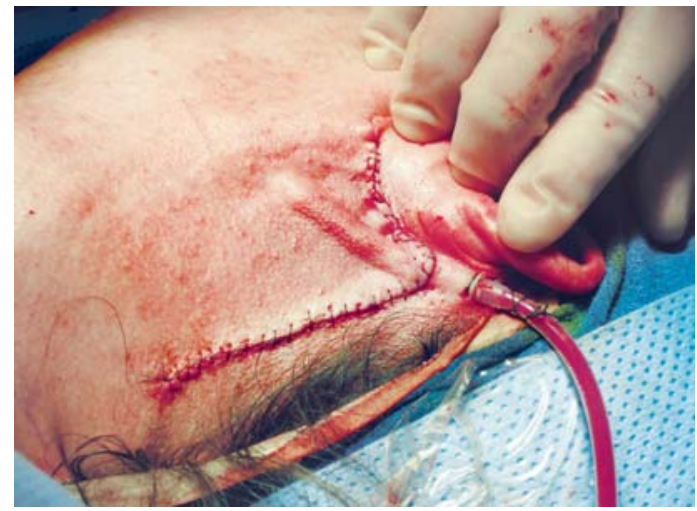

Fig. 3: Placement of the surgical drain

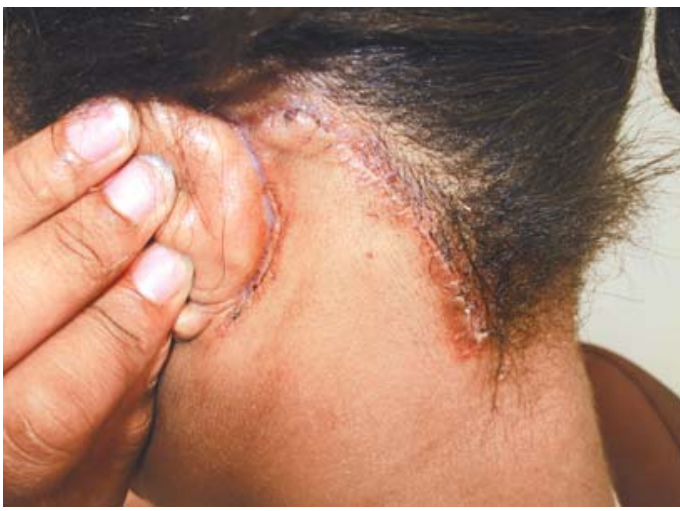

Fig. 4: Postoperative retroauricular healed incision

after obtaining an informed consent from the patient. The surgical approach is described in Table 1 (Figs 1 to 3 ).

\section{RESULTS}

\section{Case 1}

A 44-year-old female presented with a 2.5 month history of a compressible soft upper left neck mass; slowly increasing in size, without incidence of infection. CT scan showed a 
$3.8 \mathrm{~cm}$ cystic mass at the inferior margin of the left parotid gland and deep to the sternocleidomastoid muscle with minimal peripheral rim enhancement. The head and neck evaluation was otherwise unremarkable. The patient had no risk factors for head neck cancer albeit the age of presentation. The neck mass was removed via RAHI. There were no complications. Pathology was benign branchial cleft cyst.

\section{Case 2}

A 20-year-old female presented to the emergency room with complaints of a tender erythematous left upper neck mass. She was treated with antibiotics which reduced the pain and swelling associated with the neck mass. A CT scan showed a $3 \mathrm{~cm}$ left neck mass below the angle of the mandible adjacent to the anterior border of the sternocleidomastoid muscle. The neck mass was removed via RAHI. Intraoperatively, the contents of the mass were decompressed to improve surgical access. Pathology was a benign branchial cleft cyst.

\section{Complications and Cosmetic Results}

None of the patients had a postoperative infection, hematoma, spillage of cyst fluid or necrosis of the skin flap. All patients were satisfied with the cosmetic outcome of the procedure (100\%) (Fig. 4). The RAHI healed well without any evidence of hair loss or keloid formation. Complications of surgery by each case are listed in Table 2.

\section{DISCUSSION}

The RAHI has been reported to offer equivocal resection outcomes and safety, while offering a more cosmetically appealing incision for excision of upper neck masses, including branchial cleft cysts, in adults and in children. ${ }^{1,3,4}$ In a study by Roh JL et al, patients undergoing RAHI approach ranked their overall subjective satisfaction with the incision at 8.7 and 8.9 on a scale of 1 to 10 . This number is compared to an average of 4.2 subjective satisfaction score with a more traditional transcervical incision. ${ }^{7}$ In addition to the superior cosmetic outcome, RAHI offers excellent functional outcomes as well. In previous series as well as in our experience, there were no instances of marginal mandibular nerve paresis or palsy which can be a significant problem with the transcervical approach. Temporary earlobe hypoesthesia is a possible complication of this approach and should be discussed with the patients. One patient in

\begin{tabular}{lllll}
\multicolumn{5}{c}{ Table 2: Complications of RAHI approach } \\
Patient & Age & Sex & Diagnosis & Complication \\
\hline 1 & 44 & Female & Branchial cyst & None \\
2 & 20 & Female & Branchial cyst & $\begin{array}{l}\text { Temporary } \\
\text { hypoesthesia } \\
\text { of the earlobe }\end{array}$
\end{tabular}

our series had this complication which resolved spontaneously. However, in general the incidence of earlobe hypoesthesia is low. Of 20 patients analyzed, only two patients experienced temporary earlobe hypoesthesia, as well as the one patient in small case series who had similar results. ${ }^{1,3}$ Flap elevation should be undertaken with the utmost care to prevent the buttonholing of the skin. This is easily avoided with careful dissection in the appropriate surgical plane. Hypertrophic scars were not seen in our series. They have been previously reported by Roh JL et al but are well-hidden by the auricle and natural hair. ${ }^{1}$

Some potential disadvantages of the procedure include increased operative times and decreased surgical access as compared to traditional open techniques. ${ }^{7,8}$ This was reported by Roh JL et al as well as by Chen WL et al, in their individual experiences. ${ }^{3,7,8}$ In our series, the mean operative time was 110 minutes (range, 90-120 minutes). The mean operative times reported in the literature are $45 \pm$ 12 minutes $^{1}$ and 1.5 hours, ${ }^{3}$ respectively. The increased doperative times in our series are possibly related to our early experience with this technique and related surgical anatomy. The decreased surgical exposure and relatively restricted surgical view could theoretically increase risk to structures of the upper to mid neck; including the hypoglossal nerve, spinal accessory nerve, carotid artery, and internal jugular vein. This being said, there are no reported injuries to any of the above structures in the literature.

The authors who have pioneered and adopted the RAHI for removal of SBCC have contraindicated it's use for management of cystic neck metastasis from malignant disease of the head and neck and for recurrent disease. ${ }^{1,3}$ Johnson et al (2000) in a retrospective review of 121 branchial cleft cysts found an incidence of $9.9 \%$ of metastatic squamous cell carcinoma. In their analysis, patients greater than 40 years of age had a significantly higher incidence of having cystic metastasis from head and neck squamous cell carcinoma. In these patients, the authors recommended a more aggressive approach advocating an excisional biopsy with frozen section analysis and preoperative preparation for panendoscopy with biopsies of Waldeyer's ring, if frozen section reveals malignancy in all patients over 40 years of age. ${ }^{9}$ Consequently, patients at risk for head and neck cancer (age $>40$, history of alcohol and tobacco usage) and those who have preoperative cytology that suggest a possibility of cystic metastasis should be considered for a more aggressive approach. ${ }^{9}$

\section{CONCLUSION}

In conclusion, the RAHI offers an excellent surgical outcome and superior cosmetic result with no proven increased risks to the patients who require surgical excision of a benign SBCC. Since the majority of BCC are removed in young adults, age 20 to 40 , the improved cosmesis is very desirable. 


\section{ACKNOWLEDGMENTS}

The authors would like to acknowledge Professor Peter Oliver, PhD, Department of Cell Biology and Anatomy, LSU Health Science Center, New Orleans LA and David Beahm, MD, Assistant Professor, Department of Otolaryngology and Head and Neck Surgery, LSU Health Sciences Center, New Orleans, LA for their contributions.

\section{REFERENCES}

1. Roh JL, Yoon YH. Removal of pediatric branchial cleft cyst using a retroauricular hairline incision (RAHI) approach. Int J Pediatr Otorhinolaryngol 2008;72:1503-07.

2. Snissarenko EP, Kim GH, Simental AA (Jr), Zwart JE, Ransbarger DM, Kim PD. Minimally invasive video-assisted thyroidectomy: A retrospective study over two years of experience. Otolaryngol Head Neck Surg 2009;141:29-33.
3. Chen WL, Fang SL. Removal of second branchial cleft cysts using a retroauricular approach. Head Neck 2009;31:695-98.

4. Roh JL. Removal of the submandibular gland by a submental approach: A prospective, randomized, controlled study. Oral Oncol 2008;44:295-300.

5. Al-Khateeb TH, Al Zoubi F. Congenital neck masses: A descriptive retrospective study of 252 cases. J Oral Maxillofac Surg 2007;65:2242-47.

6. Acierno SP, Waldhausen JH. Congenital cervical cysts, sinuses and fistulae. Otolaryngol Clin North Am 2007;40:161-76.

7. Roh JL. Retroauricular hairline incision for removal of upper neck masses. Laryngoscope 2005;115:2161-66.

8. Roh JL. Removal of the submandibular gland by a retroauricular approach. Arch Otolaryngol Head Neck Surg 2006;132: 783-87.

9. Gourin CG, Johnson JT. Incidence of unsuspected metastases in lateral cervical cysts. Laryngoscope 2000;110:1637-41. 